\title{
Bibliocantoo
}

REPOSITÓRIO INSTITUCIONAL: POTENCIALIZANDO A VISIBILIDADE DA PRODUÇÃO CIENTIFICA DO CENTRO DE HUMANIDADES DA UFC

\author{
INSTITUTIONAL REPOSITORY: POTENTIATING THE VISIBILITY OF \\ PRODUCTION OF SCIENTIFIC HUMANITIES CENTER THE UFC
}

\author{
Lidya Nagylla de Almeida Silva ${ }^{1}$ \\ nagylla.lidya@gmail.com \\ Anizia Almeida ${ }^{2}$ \\ aniziaalmeida80@gmail.com \\ Hanna Sandy de Oliveira ${ }^{3}$ \\ nannybells@gmail.com \\ Gabriela Belmont de Farias ${ }^{4}$ \\ gabibfarias@gmail.com
}

Resumo: A gestão da informação científica é de suma importância às Instituições de Ensino Superior (IES), pois é neste ambiente que há múltiplas possibilidades de compartilhamento de ideias e desenvolvimento de novos conhecimentos, favorecendo a comunicação científica no âmbito acadêmico. $O$ presente artigo tem por objetivo apresentar as ações desenvolvidas e os desafios vivenciados pelos membros do projeto de extensão intitulado "Repositório Institucional: Preservação da Memória Científica dos Docentes do Centro de Humanidades" da Universidade Federal do Ceará. O procedimento metodológico adotado se caracteriza por ser experimental. Ao decorrer dos

\footnotetext{
${ }^{1}$ Graduanda em Biblioteconomia - UFC. Bolsista de projeto de extensão - UFC. Lattes: http://lattes.cnpq.br/7272132926500211.

${ }^{2}$ Graduanda em Biblioteconomia - UFC. Bolsista de projeto de extensão - UFC. Lattes:

${ }^{3}$ Graduanda em Biblioteconomia - UFC. Bolsista de projeto de extensão - UFC. Lattes:http://lattes.cnpq.br/7205910098441918

4 Bacharel em Biblioteconomia - UFRN. Mestra em Ciência da Informação - UFSC. Doutora em Ciência da Informação - UNESP. Docente do Departamento de Ciências da Informação e Coordenadora do Projeto de Extensão - UFC. Lattes: http://lattes.cnpq.br/9393190768810116.
} 


\section{Q Bibliocanto@}

cinco meses de funcionamento do projeto, observamos que a grande maioria dos docentes do Centro de Humanidades desconhece a resolução da instituição sobre o depósito da produção científica no repositório institucional, não possuem interatividade com o mesmo e, consequentemente, não arquivam as produções científicas. Em relação às experiências vivenciadas pelos membros do projeto verifica-se que as atividades fazem um elo entre a abordagem teórica de sala e a prática desenvolvida no projeto, permitindo uma reflexão da prática bibliotecária em relação ao gerenciamento de recursos digitais.

Palavras-chave: Informação científica. Acesso à informação. Gestão de documentos. Repositórios digitais. Repositórios institucionais.

\section{INTRODUÇÃO}

A sociedade, simultaneamente, produz e consome informação a todo o momento. De acordo com Le Coadic (2004), esse processo tem como base um ciclo de construção, comunicação e uso da informação, sendo a comunicação mediadora de todo esse processo. A informação científica, além de assegurar o contato entre os pesquisadores (ou pares), promove a comunicação das pesquisas desenvolvidas em âmbito global, regional e local, fazendo-se presente desde o início de uma pesquisa, em que a literatura do assunto será revisada pelo pesquisador, até a sua divulgação em meios formais ou informais. Sendo, portanto, imprescindível para o desenvolvimento do meio científico e tecnológico de um país. No entanto, o acesso à comunicação científica esteve, por muito tempo, enfraquecido e limitado, tomando forças e se expandido somente com a afamada "explosão informacional", decorrente da evolução das Tecnologias de Informação e Comunicação (TIC) e do reconhecimento da importância dessas comunicações para a ampliação da ciência e tecnologia. 


\section{Q Bibliocanto@}

As Instituições de Ensino Superior (IES), sendo um ambiente científico, são consideradas um berço da intensa produção de novos conhecimentos e, consequentemente, do processo de comunicação científica para possibilitar o uso dessas informações à sociedade. Para tanto, necessitam de constante atualização de recursos inovadores e seguros, favoráveis à construção, disseminação, recuperação, aplicação e transformação desses conhecimentos. Ressalvamos que o conhecimento é essencial para o ciclo de construção da comunicação científica, que, de acordo com Choo (2003, p. 36),

[...] é conseguida quando se reconhece o relacionamento sinérgico entre o conhecimento tácito e o conhecimento explícito dentro de uma organização, e quando são elaborados processos sociais capazes de criar novos conhecimentos por meio da conversão do conhecimento tácito em conhecimento explícito.

Essa conversão, que o autor se refere, necessita do amparo de uma política aliada a uma gestão que visa organizar e tratar tecnicamente o conhecimento e que reconheça a importância do processamento da informação científica para a instituição.

Diante do exposto, o presente relato de experiência objetiva apresentar um panorama das ações desenvolvidas e dos desafios vivenciados pelos membros do projeto de extensão intitulado "Repositório Institucional: Preservação da Memória Científica dos Docentes do Centro de Humanidades", cadastrado na Pró-Reitoria de Extensão da Universidade Federal do Ceará, a fim de proporcionar uma visão do compartilhamento das vivências em sala de aula com as experiências permitidas a partir das práticas realizadas no projeto para o desenvolvimento da educação e mercado de trabalho.

\section{REPOSITÓRIOS INSTITUCIONAIS: CONCEITOS}




\section{Q Bibliocanto@}

O desenvolvimento das tecnologias foi crucial para a democratização do acesso à informação, transformando vários âmbitos sociais e seus modelos de comunicação, principalmente a comunicação científica. As mudanças relacionadas ao acesso livre aos resultados de pesquisas beneficiam a comunidade acadêmica através da facilidade de comunicação entre seus membros, além do aumento da visibilidade da produção científica.

Um dos resultados do movimento de acesso livre foi a criação de Repositórios Digitais (RDs), bases de dados que têm por objetivo reunir e tornar aberto o acesso a pesquisas. Segundo Leite e Costa (2007), sua predominância em universidades possibilitou a melhoria do ensino, do aprendizado e da pesquisa, além do aumento do número de citações de autores, tornando a comunicação científica mais rápida e visível.

Os RDs podem ser divididos em temáticos, tratando da produção intelectual de uma determinada área do conhecimento; institucionais, que lidam com a produção científica de uma instituição; e de teses e dissertações, que lidam exclusivamente com teses e dissertações. (INSTITUTO BRASILEIRO DE INFORMAÇÃO EM CIÊNCIA E TECNOLOGIA, 2012).

Os Repositórios Institucionais (RIs) surgem na segunda metade de 2002, a partir do lançamento do software DSpace (desenvolvido pelo Massachussetts Institute of Technology e da Hewlett-Packard Labs, na Universidade de Cambridge, para possibilitar a criação de Rls), como uma estratégia das instituições para acompanhar as mudanças aceleradas que ocorrem na comunicação eletrônica técnico-científica e para: ser um banco de dados baseado na Web (repositório) de material escolar; ser cumulativo e perpétuo; aberto e interoperável; também para minimizar os problemas causados pelas falhas nas coleções de periódicos devido ao preço das assinaturas. (WARE, 2004). 


\section{Q Bibliocanto@}

Os Ris, de acordo com Marcondes e Sayão (2009), são bases de dados na Web na qual a instituição deposita sistematicamente sua produção científica e a disponibiliza de forma ampla para as comunidades interessadas. Os Rls são produtos informacionais que oferecem um conjunto de serviços voltados para a gestão e para a disseminação de informações em formato digital. Esses serviços incluem captura, armazenamento, tratamento técnico, organização, preservação e entrega de conteúdos digitais de toda a natureza - texto, imagem, vídeo, áudio, apresentações, programas de computador, datasets, etc. -, considerando-os como um conjunto de serviços de informação que se materializam por meio de um site Web.

Os Rls, segundo Leite (2009, p. 21), surgiram de forma inovadora no contexto da comunicação científica e do gerenciamento do conhecimento, como:

[...] um serviço de informação científica - em ambiente digital e interoperável - dedicado ao gerenciamento da produção intelectual de uma instituição. Contempla, por conseguinte, a reunião, armazenamento, organização, preservação, recuperação e, sobretudo, a ampla disseminação da informação científica produzida na instituição.

Sua estrutura permite, também, o acompanhamento dos trabalhos científicos de uma forma mais ampla e instantânea, com a disponibilidade de dados estatísticos como "número de downloads, relação downloads/citação, índices de premiações e de apoios à pesquisa por pesquisadores etc." (MARCONDES; SAYÃO, 2009). Desse modo, pesquisadores podem obter o resultado do impacto de suas pesquisas de uma maneira mais imediata do que através da espera da publicação de um periódico, por exemplo, possibilitando ampliação do diálogo entre pares e incentivando o desenvolvimento de pesquisas futuras. 


\section{Q Bibliocanto@}

Costa e Leite (2009) sustentam que os Rls possuem quatro atributos importantes: institucionalmente definido: seus limites são definidos pelas fronteiras da instituição e devem ser oficialmente reconhecidos pela instituição por meio de implementação de políticas que garantam sua existência; orientação científica e acadêmica: cientificamente orientado deve significar conteúdo reconhecido e validado pela comunidade científica e academicamente orientado flexibiliza a perspectiva da validação alcançada pelo peer review, e contemplam também outras formas de comunicação científica, mais informal, inclusive materiais de ensino; cumulativo e perpétuo: preservar a produção intelectual de uma instituição e garantir acesso amplo e irrestrito; aberto e interoperável: uma das principais características responsáveis pelo aumento do impacto dos resultados de pesquisa e visibilidade da produção, do pesquisador e da instituição.

A implantação de Repositórios, além de preservar e disponibilizar a informação científica à comunidade acadêmica, "incorpora a facilidade de comunicação, da colaboração e de outras formas de interação dinâmica entre usuários de um vasto universo" (MARCONDES; SAYÃO, 2009).

Os Rls podem também servir como representação da qualidade de uma instituição, possibilitando a transparência, pois têm o poder de "demonstrar a relevância científica, social e econômica de suas atividades de pesquisa, aumentando a visibilidade, o status e o valor público da instituição". (LEITE; COSTA 2006, p. 213).

Desse modo, é preciso pensar nas estratégias de planejamento e marketing, nas estratégias de povoamento dos RIs (aquisição de conteúdos) e nos mandatos de autoarquivamento, que constituem uma extensão do publish or perish, pelo fato de que "[...] os pesquisadores são promovidos e obtêm 


\section{Q Bibliocanto@}

financiamento com base no desempenho de pesquisa, no qual o impacto (a citação) constitui um importante indicador" (LEITE, 2009, p. 88).

\subsection{UFC: CENTRO DE HUMANIDADES E REPOSITÓRIO INSTITUCIONAL}

A Universidade Federal do Ceará (UFC) foi criada em 1954, com a Lei n. 2.373, e instalada em 1955, com a seguinte constituição: Faculdades de Direito, de Farmácia e Odontologia, de Medicina e pela Escola de Agronomia. Atualmente é dirigida pelo Prof. Dr. Henry de Holanda Campos.

Sediada em Fortaleza, Capital do Estado, a UFC é um braço do sistema do Ensino Superior do Ceará e sua atuação tem por base todo o território cearense, de forma a atender às diferentes escalas de exigências da sociedade. A Universidade Federal do Ceará é composta de sete campi, denominados Campus do Benfica, Campus do Pici e Campus do Porangabussu, todos localizados no município de Fortaleza (sede da UFC), além do Campus Avançado de Sobral, do Campus Avançado do Quixadá, Campus Avançado do Crateús e Campus Avançado de Russas. A UFC chega hoje com praticamente todas as áreas do conhecimento representadas em seus campi. (UNIVERSIDADE FEDERAL DO CEARÁ, 2016). Sua visão está em:

Consolidar-se como instituição de referência no ensino de graduação e pós-graduação (stricto e lato sensu), de preservação, geração e produção de ciência e tecnologia, e de integração com o meio, como forma de contribuir para a superação das desigualdades sociais e econômicas, por meio da promoção do desenvolvimento sustentável do Ceará, do Nordeste e do Brasil. (UNIVERSIDADE FEDERAL DO CEARÁ, 2015, p.13).

A administração acadêmica da UFC é coordenada por unidades, com a denominação de Centros, Faculdades e Institutos, sendo eles: 


\section{Bibliocantos}

1. Centro de Ciências

2. Centro de Humanidades

3. Centro de Tecnologia

4. Centro de Ciências Agrárias

5. Faculdade de Medicina

6. Faculdade de Farmácia, Odontologia e Enfermagem

7. Faculdade de Direito

8. Faculdade de Educação

9. Faculdade de Economia, Administração, Atuária, Contabilidade e Secretariado Executivo

10. Instituto de Ciências do Mar (LABOMAR)

11. Instituto de Cultura e Arte (ICA)

12. Instituto de Educação Física e Esportes (IEFES)

13. Instituto Universidade Virtual - UFC Virtual

O Centro de Humanidades $(\mathrm{CH})$ fica instalado no campus do Benfica. $\mathrm{O}$ campus formou-se, inicialmente, com a Faculdade de Filosofia, Ciências e Letras, após a Reforma Universitária da UFC, em 1969, transformou-se no Centro de Humanidades $(\mathrm{CH})$ da UFC. Após sua criação, o $\mathrm{CH}$ passou a ser constituído pela Faculdade de Ciências Sociais e Filosofia, pela Faculdade de Artes e Arquitetura e pelas Faculdades de Letras. Algum tempo depois, com a integração da Faculdade de Artes e Arquitetura ao Centro de Tecnologia, o CH deixou de ser constituído por Faculdades, tendo agora oito Departamentos e seus Cursos de graduação. Conforme apresentado na imagem abaixo: 


\begin{tabular}{|l|}
\hline Departamentos do Centro de Humanidades \\
Departamento de Ciências da Informação \\
Departamento de Ciências Sociais \\
Departamento de História \\
Departamento de Letras Estrangeiras \\
Departamento de Letras Vernáculas \\
Departamento de Letras-Libras e Estudos Surdos \\
Departamento de Literatura \\
\hline Departamento de Psicologia \\
\hline
\end{tabular}

Imagem 1 - Departamentos do CH-UFC

Fonte: http://www.ch.ufc.br/departamentos

O Centro de Humanidades "busca focar seu compromisso na solução dos problemas locais de sua área de atuação, sem esquecer o caráter universal de sua produção." (UNIVERSIDADE FEDERAL DO CEARÁ, 2016).

Assim, a fim de preservar e gerir a produção técnico-científica da Universidade de maneira mais adequada e de valorizar sua universalidade e seu potencial, o projeto RI-UFC foi aceito em 2010, através do Edital do Instituto Brasileiro de Informação em Ciência e Tecnologia (IBICT) Finep/PCAL/XBDB n. 003/2009, em que foram disponibilizados servidores e softwares para o RI, como o DSpace, atual gerenciador, a fim de apoiar e colaborar com a disseminação e o acesso à produção científica. 


\section{Bibliocantos}

Criou-se em 2011 a Resolução n.02/CONSUNI/UFC, que estabelece normas para a Política Institucional de Informação Técnico-Científica da UFC no que se refere ao seu RI.

A Resolução n.02/CONSUNI/UFC leva em consideração cinco necessidades de:

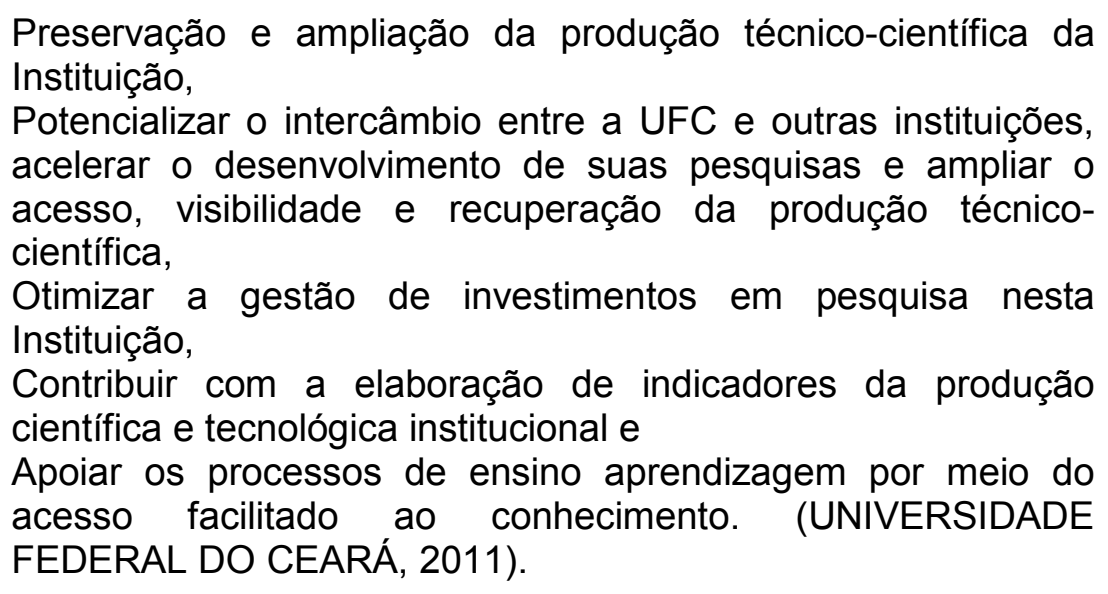

Segundo a resolução que normatiza o RI-UFC é designada a comissão de implantação e manutenção do RI por:

01 (um) representante da Pró-Reitoria de Pesquisa e Pós-Graduação;

01 (um) representante da Pró-Reitoria de Graduação;

01 (um) representante da Pró-Reitoria de Extensão;

01 (um) representante do Sistema de Bibliotecas;

01 (um) representante da Coordenadoria de Comunicação Social e Marketing Institucional;

01 (um) representante da Secretaria de Tecnologia da Informação (STI);

01 (um) representante do Diretório Central dos Estudantes (DCE).

A resolução define produção técnico-científica como sendo: 


\section{Bibliocantos}

Aquela constituída de resultados de pesquisa consolidados disponíveis em veículos de comunicação científica que tenham revisão por pares, bem como documentos produzidos, submetidos ou patrocinados pela Universidade Federal do Ceará ou por membros da comunidade universitária. (UNIVERSIDADE FEDERAL DO CEARÁ, 2011).

Para tanto, considera-se produção técnico-científica os seguintes documentos:

I - artigos publicados em periódicos científicos;

II - teses e dissertações defendidas nos programas de pósgraduação da UFC;

III - teses e dissertações defendidas extra UFC cuja autoria é de servidores desta instituição;

IV - livros e capítulos de livros;

$\mathrm{V}$ - trabalhos apresentados em eventos científicos e acadêmicos;

VI - produção cultural oriunda de trabalhos científicos e acadêmicos. (UNIVERSIDADE FEDERAL DO CEARÁ, 2011).

Atualmente, o RI possui aproximadamente 17.000 documentos disponibilizados na íntegra, de acesso livre. Estão divididos em comunidades de acordo com os campi, centros, faculdades e institutos, sendo o Centro de Humanidades a maior comunidade, contendo mais de 3.000 documentos.

\subsection{METODOLOGIA}

Este projeto de extensão nasceu após uma análise dos benefícios, apresentados na literatura, do uso do Repositório Institucional pelas instituições de pesquisa, somado à realização de um diagnóstico da adesão da comunidade docente do $\mathrm{CH}$ ao RI da UFC. O CH possui 8 departamentos, conforme apresentado na seção anterior, na qual há 221 docentes vinculados. 


\section{Q Bibliocanto@}

O diagnóstico foi realizado por meio da analise da comunidade do $\mathrm{CH}$ no RI-UFC e por uma amostragem aleatória do Currículo Lattes dos docentes, focando o item de publicação no período de 2011 a 2015.

Os procedimentos relacionados ao diagnóstico ocorreram em novembro de 2015, tendo como lapso de tempo, de publicação docente, os anos entre 2011 a 2015. É preciso ressaltar que o recorte de tempo foi determinado de acordo com o ano de criação do RI-UFC somado ao ano de elaboração da proposta do projeto. A metodologia adotada para o desenvolvimento do relato de experiência baseia-se na descrição das atividades relacionadas ao projeto.

\subsubsection{Projeto RICH-UFC}

O projeto visa o arquivamento de documentos científicos no Repositório Institucional da Universidade Federal do Ceará (RI-UFC), especificamente na comunidade do Centro de Humanidades $(\mathrm{CH})$, com o propósito de fortalecimento e visibilidade das produções científicas e tecnológicas das áreas de conhecimento que $\mathrm{O} \mathrm{CH}$ contempla. Para tanto, o projeto lida com duas perspectivas, sendo elas: sensibilizar e conscientizar da necessidade de preservar a memória científica e tecnológica dos docentes do $\mathrm{CH}$, visando potencializar a recuperação e dar visibilidade à informação científica da área de Humanas e afins; e oportunizar experiências laborais aos alunos do curso de Biblioteconomia sobre o fenômeno da tecnologia da informação e suas diversas modalidades.

O objetivo geral do projeto é o arquivamento e preservação da memória científica dos docentes do $\mathrm{CH}$, visando potencializar a recuperação e dar visibilidade à informação científica do $\mathrm{CH}$. 


\section{Q Bibliocanto@}

Tendo como objetivos específicos: a) desenvolver habilidades informacionais, por meio da prática laboral, dos bolsistas envolvidos no projeto; b) capacitar os bolsistas para realizar os procedimentos necessários até 0 arquivamento dos documentos no $\mathrm{Rl}$; c) criar um canal de comunicação com os docentes do $\mathrm{CH}$ sobre como o projeto irá funcionar além de receber documentos não localizados na web; d) arquivar toda documentação produzida pelos docentes do $\mathrm{CH}$ no período de 2011 a 2015; e) desenvolver um manual de alto arquivamento para os docentes do $\mathrm{CH}$.

\subsubsection{Desenvolvimento do Projeto RICH-UFC}

O projeto teve início em abril de 2016 e conta com o seguinte quadro de colaboradores: 9 bolsistas, sendo 4 alunos de Biblioteconomia, 2 alunos de Matemática e 1 aluno de Ciências Sociais, de História e de Letras; 4 bibliotecários do sistema de bibliotecas da UFC, sendo dois deles da Biblioteca Central (que é responsável pela gestão do sistema) e dois da Biblioteca do $\mathrm{CH}$; e uma docente do Departamento de Ciências da Informação na qual é coordenadora do projeto.

Os bolsistas juntamente com a docente tiveram quatro dias de capacitação, nos dias 25 a 28 de abril do ano de 2016, com os bibliotecários do sistema de bibliotecas da UFC, após a capacitação cada bolsista teve conhecimento do departamento que ficaria responsável em recuperar os documentos científicos e sensibilizar os docentes a aderirem ao projeto. Ficando a distribuição desta forma: 1 bolsista para o Departamento de Ciências da Informação; 1 bolsista para o Departamento de Ciências Sociais; 1 bolsista para o Departamento de História; 1 Departamento de Letras Estrangeiras; 2 bolsistas para o Departamento de Letras Vernáculas; 1 bolsista para o Departamento de Letras-Libras e Estudos Surdos; 1 bolsista para o Departamento de Literatura e 1 bolsista para o Departamento de Psicologia. 


\section{Q Bibliocanto@}

Após a capacitação e a divisão por departamentos, a atividade realizada durante o período de abril a julho de 2016 concentrou-se na identificação das produções dos docentes, tendo como fonte principal o currículo Lattes de cada um, e a recuperação e armazenamento dos documentos localizados, além das solicitações dos documentos não encontrados em ambiente web.

\section{RESULTADOS PARCIAIS}

Verificamos que a comunidade do $\mathrm{CH}$ tem um número expressivo de arquivamento no RI-UFC, entretanto não reflete significativamente a produção científica e tecnológica do corpo docente do $\mathrm{CH}$. O $\mathrm{CH}$ possui oito departamentos no qual há 221 docentes vinculados. Ao efetivar uma amostra com intuito de evidenciarmos a lacuna de arquivamento no RI-UFC, comprovamos que existe uma lacuna de arquivamento de documentos científicos na comunidade do $\mathrm{CH}$ equivalente a 2000 (dois mil) documentos. Ressaltamos que esse resultado é uma média do cálculo das produções dos docentes do $\mathrm{CH}$ registradas no currículo Lattes no período de 2011 a 2015. Com base na amostra, destacamos a relevância da necessidade de criarmos um projeto com participação de alunos para auxiliar o corpo docente no arquivamento dos documentos científicos e tecnológicos no RI-UFC, proporcionando a essa equipe vivenciar e relacionar a teoria com a prática.

Ao concluirmos o levantamento dos currículos Lattes, conforme a tabela 1, constatamos que dos 221 docentes, 7 docentes não possuem currículo na plataforma Lattes, 67 estão com o currículo Lattes desatualizado, tendo o departamento de Letras Estrangeiras o maior índice de currículos desatualizados (19), seguido do Departamento de Psicologia, com 11 currículos desatualizados. Esses indicadores demonstram que ainda muitos docentes não compreendem a real função do currículo Lattes enquanto fonte de informação. 


\section{Bibliocantos}

De acordo com a tabela 1, até o mês de julho, dos 221 docentes pertencentes ao $\mathrm{CH}$, os bolsistas efetivaram o levantamento do Lattes de 176 docentes, constatando uma demanda de 1.071 documentos não disponíveis no ambiente Web.

Tabela 1 - Levantamento do currículo Lattes e das produções dos professores

\begin{tabular}{c|c|c|c|c|c}
\hline DEPARTAMENTOS & $\begin{array}{c}\text { Total de } \\
\text { Professores }\end{array}$ & $\begin{array}{c}\text { Lattes } \\
\text { inexistent } \\
\mathbf{e}\end{array}$ & $\begin{array}{c}\text { Lattes } \\
\text { desatualizado }\end{array}$ & $\begin{array}{c}\mathbf{N}^{\circ} \text { de } \\
\text { Professores } \\
\text { (levantamento) }\end{array}$ & $\begin{array}{c}\text { Documentos } \\
\text { indisponíveis }\end{array}$ \\
\hline $\begin{array}{c}\text { Ciências da } \\
\text { Informação }\end{array}$ & 18 & 1 & 2 & 17 & 100 \\
\hline Ciências Sociais & 37 & 0 & 6 & 7 & 94 \\
\hline História & 19 & 0 & 4 & 9 & 63 \\
\hline Letras Estrangeiras & 51 & 5 & 19 & 51 & 238 \\
\hline Letras Vernáculas & 33 & 0 & 8 & 33 & 206 \\
\hline Letras-Libras & 16 & 1 & 8 & 13 & 33 \\
\hline Literatura & 16 & 0 & 9 & 16 & 153 \\
\hline Psicologia & 31 & 1 & 11 & 30 & 184 \\
\hline
\end{tabular}

Fonte: Dados do projeto, 2016.

Em relação à produtividade relacionada aos downloads dos documentos encontrados no ambiente web, ao arquivamento no RI-UFC e às correções das descrições de metadados, verificamos que foram realizados 1.181 downloads entre o mês de abril a julho, 209 arquivamentos no RI-UFC e 51 correções das descrições de metadados.

Tabela 2 - Levantamento da produtividade do projeto RICH-UFC

\begin{tabular}{c|c|c|c}
\hline BOLSISTA & ABRIL - 15/JUN & $\mathbf{1 6 - 3 0 / J U N ~}$ & $\mathbf{0 1}-\mathbf{3 1 / J U L}$ \\
\hline Anizia Almeida & 159 downloads & 18 downloads / 20 & 5 downloads / 9 submissões \\
\hline
\end{tabular}




\section{Bibliocantos}

139

\begin{tabular}{c|c|c|c}
\hline (abril) & submissões & /1 correção \\
\hline $\begin{array}{c}\text { Carliana Isabel } \\
\text { (23/maio) }\end{array}$ & 30 downloads & 3 downloads & $\begin{array}{c}16 \text { downloads / } 6 \\
\text { submissões }\end{array}$ \\
\hline $\begin{array}{c}\text { Carlos Augusto } \\
\text { (23/maio) }\end{array}$ & 15 downloads & 4 downloads / 4 submissões & $\begin{array}{c}9 \text { downloads / 13 } \\
\text { submissões }\end{array}$ \\
\hline $\begin{array}{c}\text { Charles Oliveira } \\
\text { (23/maio) }\end{array}$ & 65 downloads & $\begin{array}{c}65 \text { downloads / 29 } \\
\text { submissões }\end{array}$ & - \\
\hline $\begin{array}{c}\text { Cícera Raquel } \\
\text { (23/maio) }\end{array}$ & 71 downloads & 19 downloads / 1 submissão & $\begin{array}{c}7 \text { downloads / 12 } \\
\text { submissões }\end{array}$ \\
\hline $\begin{array}{c}\text { Hanna Sandy } \\
\text { (abril) }\end{array}$ & 172 downloads & $\begin{array}{c}120 \text { downloads / 11 } \\
\text { submissões }\end{array}$ & $\begin{array}{c}11 \text { submissões / 13 } \\
\text { correções }\end{array}$ \\
\hline $\begin{array}{c}\text { Lidya Nágylla } \\
\text { (abril) }\end{array}$ & 147 downloads & 69 submissões & $\begin{array}{c}24 \text { submissões / } 37 \\
\text { correções }\end{array}$ \\
\hline $\begin{array}{c}\text { Maria Jamile } \\
\text { (14/junho) }\end{array}$ & 1 download & 93 downloads & 11 downloads \\
\hline $\begin{array}{c}\text { Yan Bruno } \\
\text { (14/junho) }\end{array}$ & - & 79 downloads & 68 downloads \\
\hline
\end{tabular}

Fonte: Dados do projeto, 2016.

Ressaltamos que ao realizarmos a submissão dos documentos ao RI a equipe de bibliotecários responsável pelo RI-UFC avalia os metadados para liberação do documento, caso haja erro na descrição dos metadados o documento retorna para as correções necessárias.

Tabela 3 - Levantamento da produtividade do RI-UFC/CH

\begin{tabular}{c|c|c}
\hline DEPART AMENTOS & $\begin{array}{c}\text { DADOS DO RI-UFC } \\
\mathbf{0 9 / 0 5 / 2 0 1 6}\end{array}$ & $\begin{array}{c}\text { DADOS DO RI-UFC } \\
\mathbf{3 1 / 0 7 / 2 0 1 6}\end{array}$ \\
\hline Departamento de Ciências da Informação & 19 & 111 \\
\hline Departamento de Ciências Sociais & 846 & 906 \\
\hline Departamento de História & 247 & 287 \\
\hline Departamento de Letras Estrangeiras & - & 23 \\
\hline Departamento de Letras Vernáculas & 772 & 962 \\
\hline Departamento de Letras-Libras e Estudos Surdos & - & - \\
\hline Departamento de Literatura & 156 & 161 \\
\hline Departamento de Psicologia & 418 & 603 \\
\hline Total & $\mathbf{2 . 4 5 8}$ & $\mathbf{3 . 0 5 3}$ \\
\hline
\end{tabular}




\section{Q Bibliocanto@}

Fonte: Dados do projeto, 2016.

Ao analisarmos os dados apresentados na tabela 3 , verificamos que a comunidade do $\mathrm{CH}$ no RI-UFC vem tendo frutos significativos. Ressaltamos que esse fruto não se deve somente ao projeto, mas também à sensibilização da comunidade universitária da importância de estar alimentando o RI-UFC.

\section{CONSIDERAÇÕES PARCIAIS}

Observamos, na revisão da literatura, que os repositórios têm ganhado notoriedade pela facilidade de acesso ao que é produzido nas instituições. Muitos trabalhos têm sido apresentados sobre esse assunto. Os repositórios institucionais representam todo o trabalho de uma instituição. Sendo ele bem alimentado com as produções da comunidade científica, logo outros poderão surgir, como os repositórios temáticos, para mostrar em quais áreas específicas a comunidade acadêmica colabora com a produção do conhecimento.

Em relação ao objetivo geral do projeto, verificamos que está sendo alcançado o arquivamento e, consequentemente, a preservação e a visibilidade da memória científica dos docentes do $\mathrm{CH}$. Já em relação aos objetivos específicos já alcançamos totalmente dois dos cincos objetivos e dois estão em andamento, considerando que faltam cinco meses de projeto. Os objetivos alcançados totalmente estão relacionados à capacitação dos membros do projeto, que ocorreu no mês de abril do ano corrente e à criação de um canal de comunicação com os docentes por meio do e-mail do projeto. Os objetivos em andamento estão relacionados ao arquivamento das documentações produzidas pela comunidade do $\mathrm{CH}$ entre o ano de 2011 a 2015 e ao desenvolvimento das habilidades informacionais, por meio da prática laboral, 


\section{Q Bibliocanto@}

dos bolsistas envolvidos no projeto. O manual de autoarquivamento para os docentes do $\mathrm{CH}$ será desenvolvido em novembro para ser apresentado ao sistema de Bibliotecas da UFC.

Por fim, considera-se que o projeto vem proporcionando aos seus membros uma visão diferenciada relacionada ao universo de suas práticas laborais e do que tange as fontes de informação existentes no ambiente acadêmico. Muitas descobertas e curiosidades surgem por meio do funcionamento do projeto, enriquecendo a compreensão e o funcionamento do RI.

Abstract: The management of scientific information is of paramount importance to higher education institutions, it is in this environment that there are multiple ideas sharing possibilities and developing new knowledge, promoting scientific communication in the academic field. This article aims to present the actions developed and the challenges experienced by members of the extension project titled - "Institutional Repository: Scientific Memory Preservation of Humanities Center Faculty" of the Federal University of Ceará. The methodological procedure adopted is characterized by being experimental. In the course of five months of operation of the project, we found that the vast majority of teachers of the Humanities Center, unaware of the resolution of the institution on the deposit of scientific production in institutional repository, have no interaction with the institutional repository consequently not archive the productions scientific. Regarding the experiences lived by the members of the project, it appears that the activities make a link between the theoretical approach room with the practice developed in the project, allowing a reflection of practice librarian in relation to the management of digital resources.

Keywords: Scientific information. Access to information. Document management. Digital repositories. Institutional repositories. 


\section{(2) Bibliocantos}

\section{REFERÊNCIAS}

$\mathrm{CHOO}$, Chun Wei. Preenchendo as lacunas cognitivas: como as pessoas processam informações. In: DAVENPORT, Thomas H.; MARCHAND, Donald, A.; DICKSON, Tim. Dominando a gestão da informação. Porto Alegre: Bookman, 2004. p.1279-1287.

COSTA, Sely Maria de Souza; LEITE, Fernando César Lima. Insumos conceituais e práticos para iniciativas de repositórios institucionais de acesso aberto à informação científica em bibliotecas de pesquisa. In: SAYÃO, Luis Fernando et al. (Org.). Implantação e gestão de repositórios institucionais: políticas, memória, livre acesso e preservação. Salvador: EDUFBA, 2009.

INSTITUTO BRASILEIRO DE INFORMAÇÃO EM CIÊNCIA E TECNOLOGIA. Repositórios digitais. 2012. Disponível em: $<$ http://www.ibict.br/informacaopara-ciencia-tecnologia-e-inovacao\%20/repositorios-digitais/repositoriosbrasileiros> Acesso em: 30 jul. de 2016.

LE COADIC, Y. F. Princípios científicos que direcionam a ciência e a tecnologia da informação digital. Transinformação, Campinas, v. 16, n. 3, p. 205-213, set./ dez. 2004.

LEITE, F. C. L.. Como gerenciar e ampliar a visibilidade da informação científica brasileira: repositórios institucionais de acesso aberto. Brasília: IBICT, 2009.

LEITE, F. C. L.; COSTA. S. Repositórios institucionais como ferramentas de gestão do conhecimento científico no ambiente acadêmico. Perspectiva Ciência da Informação, Belo Horizonte, v.11 n. 2, p. 206-219, maio/ago. 2006.

. Gestão do conhecimento científico: proposta de um modelo conceitual com base em processos de comunicação científica. Ci. Inf. [online], v. 36, n.1, p.92-107, 2007. ISSN 0100-1965. Disponível em: <http://dx.doi.org/10.1590/S0100-19652007000100007>. Acesso em: 3 ago. 2016.

MARCONDES, Carlos Henrique; SAYÃO, Luis Fernando. À guisa de introdução: repositórios institucionais e livre acesso. In: SAYÃO, Luis Fernando et al. (Org.). Implantação e gestão de repositórios institucionais: políticas, memória, livre acesso e preservação. Salvador: EDUFBA, 2009. p. 9-2. 


\section{- Bibliocantos}

REPOSITÓRIO Institucional UFC. Estatísticas. Disponível em: <http://www. repositorio.ufc.br/handle/riufc/232/statistics>. Acesso em: 21 jul. 2016.

UNIVERSIDADE FEDERAL DO CEARÁ. Resolução Nº 02/CONSUNI, de 2011. Estabelece normas para a Política Institucional de Informação TécnicoCientífica da UFC no que se refere ao seu Repositório Institucional (RI). Fortaleza, CE, 29 de abril de 2011.

A Universidade - Início. Disponível em: <http://www.ufc.br/auniversidade>. Acesso em: 3 ago. 2016.

Anuário Estátistico Universidade 2015 - base 2014. Disponível em: <http://www.ufc.br/images/_files/a_universidade/anuario_estatistico/anuario_est atistico_ufc_2015_base_20114.pdf > . Acesso em: 3 ago. 2016.

Repositório Institucional da UFC disponibiliza acervo digital com mais de 7.200 documentos. 2014. Disponível em:

<http://www.ufc.br/noticias/noticias-de-2014/5231-repositorio-institucional-daufc-disponibiliza-acervo-digital-com-mais-de-7-200-documentos>. Acesso em: 3 ago. 2016.

Sobre o Centro de Humanidades. Disponível em:

<http://www.ch.ufc.br /sobre-o-centro-de-humanidades/>. Acesso em: 3 ago. 2016.

WARE, Mark. Institutional repositories and scholarly publishing. Learned Publishing, v. 17, n.2, p. 115-124, 2004. 